\title{
Carcinoembryonic Antigen in Bronchoalveolar Lavage Fluid in Patients with Idiopathic Pulmonary Fibrosis
}

\author{
Hideki Takahashi, MD, Toshihiro Nukiwa, MD, Rokuro Matsuoka, MD, \\ Takashi Danbara, MD, Hiroshi Natori, MD, Tatsuo Arai, MD \\ and Shiro KIRA, MD
}

\begin{abstract}
An increased incidence of lung cancer and epithelial metaplasia or hyperplasia which is felt to be as a precursor of cancer, has been reported in patients with idiopathic pulmonary fibrosis (IPF). In this study, carcinoembryonic antigen (CEA) in bronchoalveolar lavage (BAL) fluid was measured in 53 control patients, 31 patients with sarcoidosis, 10 patients with hypersensitivity pneumonitis, 16 patients with primary lung cancer and 26 patients with histologically confirmed IPF. High ratio of CEA to albumin (Alb), exceeding mean $+2 \mathrm{SD}$ of nonsmoking control patients, were found in $8(25 \%)$ out of $32 \mathrm{smoking}$ control patients, $4(44 \%)$ out of 9 nonsmoking patients with IPF, $8(62 \%)$ out of 13 smoking patients with IPF, $3(75 \%)$ out of 4 smoking patients with IPF and lung cancer and $13(81 \%)$ out of 16 patients with primary lung cancer, although BAL was performed at the noncancerous parts of the lung in the cases of lung cancer. Furthermore, it was confirmed that CEA increased in BAL fluid in these subjects were different from nonspecific cross-reacting antigen (NCA) which was detectable in the normal lung. Thus we consider that the increase of CEA/Alb ratio in BAL fluid is a possible marker of these early histological disorders in the lung, and also suggests a greater risk of malignant change in the clinical course of IPF.
\end{abstract}

Key Words: Carcinoembryonic antigen (CEA), Idiopathic pulmonary fibrosis (IPF), Primary lung cancer, Bronchoalveolar lavage (BAL)

Patients with idiopathic pulmonary fibrosis (IPF) have been reported to have a higher incidence of lung cancer than expected ${ }^{1-3}$. In patients with IPF, epithelial metaplasia or hyperplasia is frequently found in the lower respiratory tract by postmortem examinations, and may closely relate to the high incidence of the cancer ${ }^{2,3)}$.

It is also well known that carcinoembryonic antigen (CEA) is a cancer related antigen, and is frequently high in the serum of patients with various malignant diseases ${ }^{4}$. Blair and Goldenberg analyzed the CEA content of bronchial washings, and Lemarie and co-workers measured the CEA in bronchoalveolar lavage (BAL) fluid of patients with lung cancer ${ }^{5,6)}$. These investigators noted an increase of the CEA in the lung lining fluid recovered from subjects with lung cancer. Recently, Merill and his colleagues reported a significant increase of the ratio of CEA to total protein in BAL fluid recovered from a subgroup of normal smokers and they speculated that an increase of CEA in BAL fluid may be an indicator of airway injury and metaplasia ${ }^{7)}$.

Based on these observation, we measured CEA in BAL fluid and serum of patients with IPF in comparison with those of control patients and the patients with sarcoidosis, hypersensitivity pneumonitis and primary lung cancer.

From Division of Respiratory Diseases, Department of Medicine, Jichi Medical School, Tochigi, Japan. Received for publication June 3, 1985.

Reprints request to: Hideki Takahashi, MD. Division of Respiratory Diseases, Department of Medicine, Jichi Medical School, Minamikawachi-machi, Kawachi-gun, Tochigi-ken 329-04, Japan. 


\section{METHODS}

Subjects: The subjects in this study were composed of 53 control patients, 31 patients with sarcoidosis, 10 patients with hypersensitivity pneumonitis, 26 patients with IPF and 16 patients with primary lung cancer. The consent for this study was obtained from all patients.

The control patients had no abnormal findings on their chest X-ray films, but were bronchoscopically studied because of bloody sputum or chronic cough. Twenty-one of the control patients were nonsmokers (age; $46.0 \pm 3.0$ years, mean \pm SEM: 4 males, 17 females) and 32 were smokers (45.4 \pm 2.4 yr: 27 males, 5 females).

The diagnosis of sarcoidosis was established by clinical picture (bilateral hilar lymphadenopathy and/or pulmonary infiltrates on the chest $\mathrm{X}$-ray, high value of serum angiotensin converting enzyme activity and systemic symptoms such as uveitis, dermatologic lesions, superficial lymphadenopathy etc.) and by transbronchial lung biopsy demonstrating noncaseating epithelioid cell granuloma. In patients with sarcoidosis, 25 were nonsmokers $(40.4 \pm 3.0 \mathrm{yr}: 1$ male, 24 females) and 6 were smokers $(24.7 \pm 1.8 \mathrm{yr}: 3$ males, 3 females).

The diagnosis of hypersensitivity pneumonitis was established by clinical picture (dry cough, dyspea, fever and crepitant rales relating to antigen exposure, bilateral small nodular shadows on the chest X-ray) and by transbronchial lung biopsy demonstrating noncaseous granuloma and alveolitis with infiltration of lymphocytes, plasma cells and histiocytes. In patients with hypersensitivity pneumonitis, 8 were nonsmokers $(37.6 \pm 5.3 \mathrm{yr}$ : all females) and 2 were smokers $(45.0 \pm 6.0 \mathrm{yr}$ : all males).

The diagnosis of IPF was established by clinical criteria (persistent and/or progressive dyspnea, widespread persisting crackles, widespread bilateral interstitial shadow on the chest X-ray and restrictive impairment of pulmonary function tests) and by histological findings obtained by transbronchial lung biopsy (17 cases) or autopsy (9 cases). Inhalation of organic or inorganic dusts, collagen vascular diseases, and other known causes had been carefully excluded. In patients with IPF, 9 were nonsmokers $(62.0 \pm 1.4 \mathrm{yr}$ :
1 male, 8 females) and 17 were smokers $(61.4 \pm$ 1.8 yr: 14 males, 3 females). Four out of 17 smoking patients with IPF were complicated with lung cancer, 2 cases of small cell carcinoma and 2 cases of adenocarcinoma.

The diagnosis of primary lung cancer was confirmed by bronchial mucosal biopsy and/or brushing through the fiberoptic bronchoscope. The histological type of sixteen patients was classified as epidermoid carcinoma in 9 patients, adenocarcinoma in 3 and small cell carcinoma in 4 .

No patients were treated with corticosteroid or immunosuppressive agents at the time of bronchoalveolar lavage.

Bronchoalveolar lavage: In all patients, BAL was carried out with $150 \mathrm{ml}$ of physiologic saline in a segment or subsegment of the right upper anterior bronchus. In patients with IPF and primary lung cancer, by the findings of chest Xray, fiberoptic bronchoscope and the cytological examinations of BAL fluid, it was confirmed that the segment submitted to BAL was free from the cancerous change. Recovered fluid was centrifuged at $400 \mathrm{~g}$ for $5 \mathrm{~min}$ and supernatant was stored at $-70^{\circ} \mathrm{C}$ until analyzed. Samples of serum were obtained at the time of $\mathrm{BAL}$ and stored at $-70^{\circ} \mathrm{C}$ as well.

Cellular analysis of BAL fluid: Cell number was determined by hemocytometer counts of the BAL fluid. Morphologic characteristics of the recovered cell were determined from WrightGiemsa stained cytocentrifuge cell monolayers.

CEA measurement: CEA was measured by an enzyme immunoassay based on "sandwich principle" with a highly specific monoclonal antibody (Hoffman La Roche Inc., Basel, Switzerland). Briefly, $0.05 \mathrm{ml}$ of specimen or CEA standard, $0.2 \mathrm{ml}$ of peroxidase conjugated anti-CEA antibody and polystyrene beads coated with monoclonal anti-CEA antibody were incubated in a test tube at $37^{\circ} \mathrm{C}$ for 16 to 24 hours. After that, the beads were washed 5 times with distilled water and the enzyme activity was measured. CEA in BAL fluid could be detected in all subjects by this method without any concentration. To compare the amounts of CEA in BAL fluid among respective study groups, CEA in BAL fluid are given relative to albumin of BAL fluid $(\mathrm{ng} / \mathrm{mg})$. 
Simultaneously, CEA in serum was measured by the same method. Total protein was measured by the method of Lowry and co-workers with bovine serum albumin as the reference standard ${ }^{8)}$. Albumin was measured by single radial immunodiffusion (Behringwerke AG, West Germany).

Sephadex G-200 chromatography: It is reported that normal lung tissue contains a large amounts of nonspecific cross-reacting antigen $(\mathrm{NCA})^{9,10)}$, which has a similar amino acid sequence as $\mathrm{CEA}^{11)}$. Therefore, it is necessary to exclude a possibility that we measured NCA even with the monoclonal antibody to CEA used in the present study. Thus, we tried to discriminate CEA from NCA in BAL fluid by Sephadex G-200 chromatography, since the molecular weight of NCA in normal lung tissue is estimated to be about 80,000 to 90,000 , and that of CEA to be about $180,000^{12)}$.

The low speed supernatant fraction of BAL fluid was concentrated to approximately one tenth of the original volume in an Amicon concentration cell fitted with UM-2 membrane (Amicon Far East Ltd., Tokyo, Japan). Samples of serum or concentrated BAL fluid were centrifuged at $11,000 \mathrm{~g}$ for $60 \mathrm{~min}$, mixed with equal volume of elution buffer and applied to the
Sephadex G-200 (Pharmacia Fine Chemicals, Uppsala, Sweden) chromatography. Sephadex G-200 chromatography was performed on a $2.5 \times 100 \mathrm{~cm}$ column in $0.1 \mathrm{M}$ Tris- $\mathrm{HCl}$ buffer, pH 8.00 containing $1 \mathrm{M} \mathrm{NaCl}$. An upward flow at a rate of $15 \mathrm{ml} /$ hour was maintained by Gilson's Uni-pulse 2 pump. Fractions of $8 \mathrm{ml}$ were collected by a fraction collector. The procedures were carried out at $4^{\circ} \mathrm{C}$. Protein was measured in the chromatographic fraction by photometry at $280 \mathrm{~nm}$. CEA in respective fractions was analyzed with the same method as used in BAL fluid and serum studies.

All statistical comparisons were made using the two tailed $t$ test.

\section{RESULTS}

Analysis of cellular components and the concentration of albumin in BAL fluid (Table 1): Total cell number in BAL fluid was significantly increased in smoking control patients, patients with sarcoidosis (nonsmokers, smokers), hypersensitivity pneumonitis (nonsmokers, smokers), IPF (nonsmokers, smokers) and slightly increased in the patients with primary lung cancer (smokers) compared to the nonsmoking control patients. The percentage of lymphocytes in BAL fluid

Table 1. Cell components and albumin concentration in lavage fluid

\begin{tabular}{|c|c|c|c|c|c|c|c|}
\hline & & $\begin{array}{c}\text { Recovery } \\
(\%)\end{array}$ & $\begin{array}{l}\text { Total Cells } \\
\qquad\left(\times 10^{6}\right)\end{array}$ & $\begin{array}{c}\text { Albeolar } \\
\text { Macrophage } \\
\text { (\%) }\end{array}$ & $\begin{array}{c}\text { Lymphocyte } \\
\text { (\%) }\end{array}$ & $\begin{array}{l}\text { Polymorpho- } \\
\text { nuclear } \\
\text { Leukocyte } \\
(\%)\end{array}$ & $\begin{array}{c}\text { Albumin } \\
\text { Concentration } \\
(\mathrm{ng} / \mathrm{ml})\end{array}$ \\
\hline \multirow{2}{*}{ Control Patients } & NS (21) & $53.5 \pm 2.4$ & $15.6 \pm 1.7$ & $89.8 \pm 1.3$ & $7.8 \pm 0.9$ & $1.5 \pm 0.3$ & $0.10 \pm 0.01$ \\
\hline & $\mathrm{S} \quad(32)$ & $49.9 \pm 2.6$ & $30.0 \pm 3.3^{* *}$ & $90.2 \pm 2.8$ & $6.1 \pm 0.8$ & $0.9 \pm 0.2$ & $0.10 \pm 0.01$ \\
\hline \multirow{2}{*}{ Sarcoidosis } & NS (25) & $58.5 \pm 2.1$ & $26.5 \pm 3.0^{* *}$ & $67.7 \pm 3.8^{\#}$ & $31.3 \pm 3.8^{\#}$ & $1.2 \pm 0.4$ & $0.19 \pm 0.03^{* *}$ \\
\hline & $S(6)$ & $53.6 \pm 2.7$ & $37.7 \pm 6.6 \#$ & $85.3 \pm 4.9$ & $14.1 \pm 4.7^{*}$ & $0.6 \pm 0.2$ & $0.14 \pm 0.03$ \\
\hline Hypersensitivity & NS ( 8) & $55.7 \pm 5.6$ & $61.4 \pm 14.7 \#$ & $22.5 \pm 2.7 \#$ & $72.6 \pm 2.4 \#$ & $4.9 \pm 1.9 *$ & $0.36 \pm 0.12^{* *}$ \\
\hline Pneumonitis & $S \quad(2)$ & $59.5 \pm 4.5$ & $87.1 \pm 32.9 \#$ & $27.2 \pm 9.8 \#$ & $70.9 \pm 10.1 \#$ & $3.9 \pm 2.2$ & $0.59 \pm 0.44 \#$ \\
\hline \multirow{2}{*}{ IPF } & NS ( 9) & $44.3 \pm 5.5 *$ & $41.1 \pm 3.8^{\#}$ & $75.1 \pm 3.8^{* *}$ & $14.2 \pm 3.8^{*}$ & $9.6 \pm 2.1 \#$ & $0.16 \pm 0.04 *$ \\
\hline & S (17) & $43.6 \pm 2.7 *$ & $33.0 \pm 5.7^{* *}$ & $82.6 \pm 2.6^{*}$ & $8.7 \pm 2.0$ & $8.4 \pm 1.8^{\#}$ & $0.12 \pm 0.01$ \\
\hline $\begin{array}{l}\text { Primary Lung } \\
\text { Cancer }\end{array}$ & $S \quad(16)$ & $48.6 \pm 3.1$ & $21.4 \pm 2.2^{*}$ & $75.2 \pm 6.3^{*}$ & $9.4 \pm 2.0$ & $10.0 \pm 2.6^{\#}$ & $0.09 \pm 0.03$ \\
\hline
\end{tabular}

Table 1. Values are the mean \pm SEM. Control patients means patients with bloody sputum or chronic cough, but with normal chest $\mathrm{X}$-ray. Definition of abbreviations: $\mathrm{IPF}=$ idiopathic pulmonary fibrosis, NS = nonsmoker, $\mathrm{S}=\mathrm{smoker}$.

${ }^{*} \mathrm{p}<0.05$ compared with the nonsmoking control patients.

$* * p<0.01$ compared with the nonsmoking control patients.

$\#_{p}<0.001$ compared witht he nonsmoking control patients. 
was markedly increased in nonsmoking patients with sarcoidosis and hypersensitivity pneumonitis (nonsmokers, smokers), and slightly elevated in smoking patients with sarcoidosis and nonsmoking patients with IPF compared to control patients.

The percentage of polymorphonuclear leucocytes in BAL fluid was approximately $1.0 \%$ in control patients and patients with sarcoidosis. In nonsmoking patients with hypersensitivity pneumonitis, the percentage of polymorphonuclear leukocytes were slightly higher than that of control patients $(\mathrm{p}<0.05)$. In comparison with controls and patients with sarcoidosis, patients with IPF (nonsmokers, smokers) and primary lung cancer had a significantly elevated proportion of polymorphonuclear leucocytes ( $p$ $<0.001)$.

The concentration of albumin in BAL fluid was increased in nonsmoking patients with sarcoidosis, patients with hypersensitivity pneumonitis (nonsmokers, smokers) and IPF (nonsmokers) compared to the control patients.

CEA analysis in BAL fluid (Fig. 1): The CEA to albumin (Alb) ratios in BAL fluid are shown in Fig. 1. In control patients, nonsmokers averaged

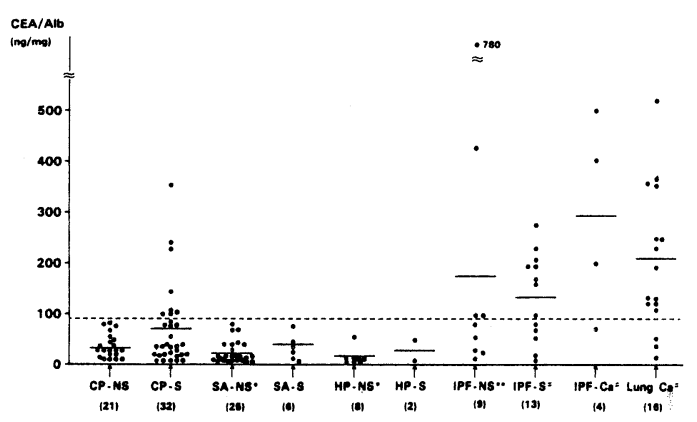

Fig. 1. The ratio of carcinoembryonic antigen to albumin (CEA/Alb, ng/mg) in BAL fluid in control patients (CP), patients with sarcoidosis (SA), hypersensitivity pneumonitis (HP), idiopathic pulmonary fibrosis (IPF) and primary lung cancer (Lung ca). Control patients, patients with sarcoidosis and hypersensitivity pneumonitis were divided into nonsmokers (NS) and smokers (S). Patients with IPF were divided into nonsmokers (NS), smokers (S) and smokers with lung cancer (IPF-Ca). All patients with primary lung cancer were smokers. Means are represented by horizontal bars. Horizontal broken line represents mean $+2 \mathrm{SD}$ of nonsmoking control patients. ${ }^{*} \mathrm{p}<0.05, *{ }^{*} \mathrm{p}<0.02$, $\#_{\mathrm{p}}<0.001$ compared with nonsmoking control patients.
$37.0 \pm 5.5$ (mean \pm SEM, ng/mg), and smokers, $71.2 \pm 13.8$. There was no significant difference between these two groups, but in 8 cases out of 32 smokers, the CEA/Alb ratio exceeded mean $+2 \mathrm{SD}(88 \mathrm{ng} / \mathrm{mg})$ from those of nonsmoking control patients, represented by the horizontal broken line.

The average CEA/Alb ratio in nonsmoking patients with sarcoidosis was $22.3 \pm 5.0$, smoking patients with sarcoidosis, $47.2 \pm 23.4$, nonsmoking patients with hypersensitivity pneumonitis, $16.4 \pm 5.6$ and smoking patients with hypersensitivity pneumonitis, $47.2 \pm 23.4$, respectively. The $\mathrm{CEA} / \mathrm{Alb}$ ratio of nonsmoking patients with sarcoidosis and hypersensitivity pneumonitis were slightly lower than that of control patients $(p<$ 0.05 ). However, this result seemed to be due to higher values of albumin concentration in $\mathrm{BAL}$ fluid in these patients compared to the control patients.

Twenty-six patients with IPF were divided into three groups, namely, 9 cases of nonsmokers, 13 of smokers without lung cancer and 4 of smokers with lung cancer. The average CEA/ Alb ratio of nonsmoking patients with IPF was $176 \pm 86.7$, that of the smokers without lung cancer, $130 \pm 23.4$ and that of the smokers with lung cancer, $292 \pm 98.0$, respectively. The CEA/ Alb ratios in BAL fluid of IPF, especially of those with lung cancer, were markedly higher than in the nonsmoking control patients (for nonsmoking IPF patients, $\mathrm{p}<0.02$, for smoking IPF patients with or without lung cancer, $p<0.001$ ). In 4 out of 9 IPF nonsmokers, 8 out of 13 IPF smokers without lung cancer and 3 out of 4 IPF patients with lung cancer, the CEA/Alb ratios exceeded 2SD above the mean of the nonsmoking control patients.

The average of CEA/Alb ratio in sixteen patients with primary lung cancer was $209 \pm 33.2$ and also significantly higher than that of nonsmoking control patients $(p<0.001)$. In 13 out of 16 patients, CEA/Alb ratios exceeded 2SD above the mean of the nonsmoking control patients.

There was no correlation between the CEA/ Alb ratio in BAL fluid and smoking history in pack-years, patients' age, sex, percentage of 
polymorphonuclear leukocytes or lymphocytes in BAL fluid.

Since the previous investigators used total protein (TP) as a lavage standard to compare the values of CEA in BAL fluid (e.g. CEA to TP ratio) ${ }^{7)}$, we also studied CEA/TP ratio in our subjects. Although the values of CEA/TP ratio were smaller than those of CEA/Alb ratio, the ultimate results were similar to those of CEA/ Alb ratio (data not shown).

CEA analysis in serum (Fig. 2): Serum CEA values $(\mathrm{ng} / \mathrm{ml})$ of control patients, patients with sarcoidosis, hypersensitivity pneumonitis, IPF with or without lung cancer and primary lung cancer were shown in Fig. 2. The average for control patients was $2.0 \pm 0.2$, for sarcoidosis, $1.5 \pm 0.3$, for hypersensistivity pneumonitis, $1.7 \pm 0.2$, for IPF without lung cancer, $5.6 \pm 0.9$, for IPF with lung cancer, $11.6 \pm 9.2$ and for primary lung cancer, 6:6 \pm 2.0 , respectively. There were no significant differences between

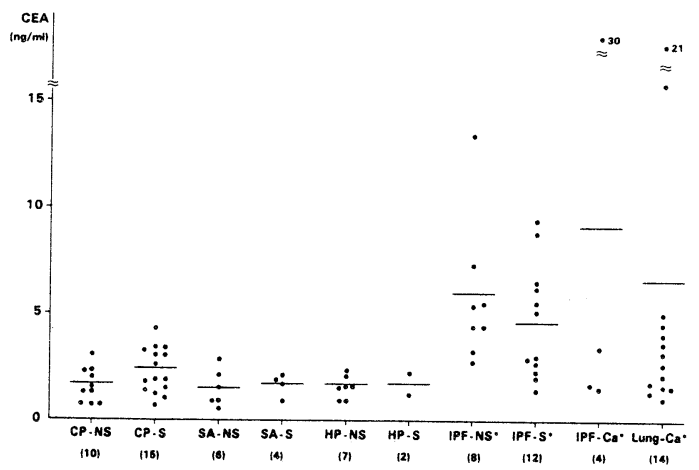

Fig. 2. The concentration of carcinoembryonic antigen (CEA, $\mathrm{ng} / \mathrm{ml}$ ) in serum in control patients (CP), patients with sarcoidosis (SA), hypersensitivity pneumonitis (HP), idiopathic pulmonary fibrosis (IPF) and primary lung cancer (Lung $\mathrm{Ca}$ ). Control patients, patients with sarcoidosis and hypersensitivity pneumonitis were divided into nonsmokers (NS) and smokers (S). Patients with IPF were divided into nonsmokers (NS), smokers (S) and smokers with lung cancer (IPF$\mathrm{Ca})$. All patients with primary lung cancer were smokers. Means are represented by horizontal bars. Samples of serum were obtained from 25 out of 53 control patients, 10 of 31 patients with sarcoidosis, 9 of 10 patients with hypersensitivity pneumonitis, 24 of 26 patients with IPF and 14 out of 16 patients with primary lung cancer at the time of BAL. ${ }^{*} \mathrm{p}<0.05$ compared with control patients. nonsmokers and smokers in each disease groups. Although serum CEA was significantly higher in IPF patients with or without lung cancer and patients with primary lung cancer in comparison with the control and sarcoidosis patients $(\mathrm{p}<$ 0.05 , each comparison), serum $\mathrm{CEA} / \mathrm{Alb}$ ratios were remarkably lower than that of BAL fluid, ranging from 0.02 to $1.2 \mathrm{ng} / \mathrm{mg}$. No correlation was detected in CEA/Alb ratio between serum

SEPHADEX-G-200 CHROMATOGRAPHY OF SERUM OBTAINED FROM PATIENT WITH LUNG CANCER

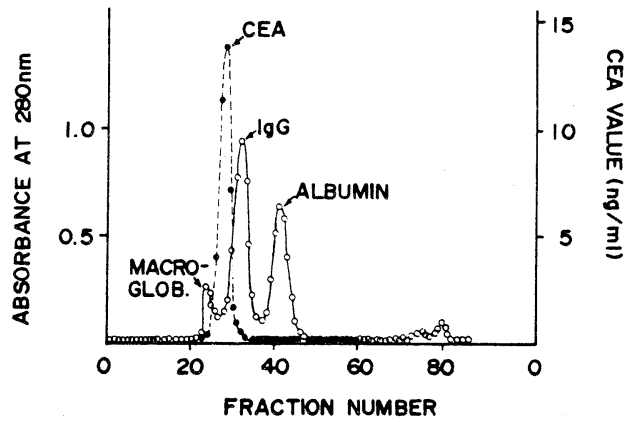

Fig. 3a. Sephadex G-200 chromatography of serum obtained from a patient with primary lung cancer on a $2.5 \times 100 \mathrm{~cm}$ column. Fraction of $8 \mathrm{ml}$ were collected. CEA was measured from fraction 21 to fraction 60 . $\circ$, absorbance at $280 \mathrm{~nm}$, •, concentration of CEA. Arrows indicate position of elution of $\alpha$ 2-macroglobulin, CEA, Ig-G and albumin. Fractions of gamma-globulin were larger than those of albumin, because the patient also had liver cirrhosis.

SEPHADEX - G - 200 CHROMATOGRAPHY OF BAL FLUID RECOVERED FROM PATIENT WITH IPF

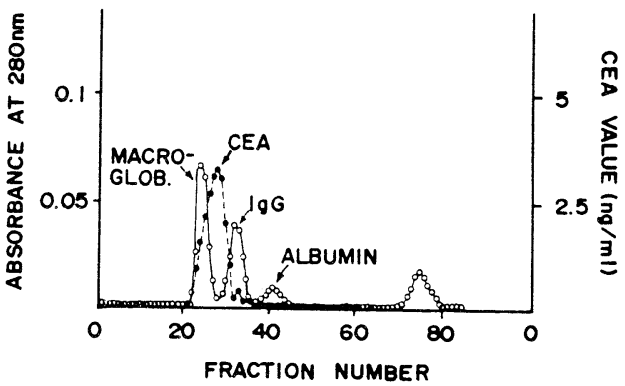

Fig. 3b. Sephadex G-200 chromatography of concentrated BAL fluid recovered from a nonsmoking patient with IPF. The peak of CEA was detected at a similar position to that of serum CEA shown in Fig. 3a. 
and BAL fluid in any of the subjects in the present study.

Sephadex G-200 chromatography analysis (Fig. 3): Fig. 3a shows Sephadex G-200 chromatography of the serum from a patient with lung cancer, who had specially high serum CEA value. An elution pattern revealed three protein peaks. The first peak consisted of marcromolecular glubulins, the second peak, Ig-G and the third peak, mainly albumin. CEA measured with the monoclonal antibody used in the present study, was eluted between the first and second peak, therefore the molecular weight of CEA is estimated to be greater than 160,000 . Since CEA contains considerable amounts of polysaccharide, we could not determine the precise molecular weight of CEA by the gel chromatography.

Fig. 3b shows Sephadex G-200 chromatography of concentrated BAL fluid from a nonsmoking patient with IPF. The peak of the CEA was almost identical with that of the serum CEA, shown in Fig. 3a. Similar results were observed in a smoking control patient and in a patient with alveolar cell carcinoma, who showed high CEA values in their BAL fluid.

\section{DISCUSSION}

There are many reports suggesting a high incidence of lung cancer in IPF and other pulmonary fibrosis ${ }^{1-3)}$. Spain reported twelve cases of terminal bronchiolar carcinoma associated with interstitial inflammation and fibrosis of the lung ${ }^{13)}$. Meyer and Liebow found $32(22 \%)$ patients with lung cancer associated with honeycombing and atypical epithelial proliferation in their series of 153 resected lung tumors and also observed transitions from atypical epithelial proliferation to obvious neoplastic change ${ }^{14)}$. In these reports and others, atypical epithelial proliferation or metaplasia in the lower respiratory tract were postulated to precede the development of invasive cancer $2,3,13,14)$.

We found 18 (54\%) patients of lung cancer out of 40 patients with IPF, who had been admitted to our department from 1975 to 1984 . The diagnosis of IPF was confirmed by transbronchial lung biopsy (TBLB) in 18 patients, autopsy in 13, TBLB and autopsy in 7 , and open lung biopsy and autopsy in 2. Moreover, 5 cases out of 18 patients with IPF and lung cancer had double cancer of the lung. Epithelial metaplasia or hyperplasia in the lower respiratory tract was also observed in all patients with IPF in the postmortem examinations. An epidemiologic study was not done, but keeping in mind that the numbers of patients with lung cancer may reflect one patterns of referral, the incidence of lung cancer in the IPF seemed to be markedly high in comparison with the other types of interstitial lung disease.

Because it is well-known that CEA is one of the cancer related antigens, and is frequently high in the serum of patients with various malignant diseases ${ }^{4}$, a great deal of attention is focused on CEA in BAL fluid in these patients and individuals who are in high risk to lung cancer.

An increase in the amount of CEA recovered from the cancerous lung was reported by Blair and Goldenberg, who studied with bronchial washing ${ }^{5)}$. A similar result was confirmed by Lamarie and his colleagues who studied with BAL fluid ${ }^{6)}$. Merill and his colleagues described a significant increase of the CEA/TP ratio in BAL fluid recovered from a subgroup of normal smokers. They suggested that the increase of CEA in lung lining fluid may be an indicator of airway injury and metaplasia in a subsegment of the smoking population ${ }^{\text {?) }}$.

In the present study, we compared CEA in BAL fluid and serum obtained from IPF patients who are known to have a high incidence of lung cancer as described above, with the control patients and patients with sarcoidosis, hypersensitivity pneumonitis and primary lung cancer.

As reported by the previous investigators ${ }^{5-7)}$, high values of CEA/Alb ratios in BAL fluid were obtained in a subgroup of the smoking control patients and patients with primary lung cancer, although BAL was performed at noncancerous parts of the lung in the cases of lung cancer. In $8(25 \%)$ out of 32 smoking control patients and $13(81 \%)$ out of 16 patients with primary lung cancer, CEA/Alb ratios in BAL fluid exceeded the mean + 2SD from those of nonsmoking control patients.

Furthermore, significantly higher values of 
CEA/Alb ratios in BAL fluid were also observed in patients with IPF, namely, in $4(44 \%)$ out of 9 nonsmoking patients with IPF, $8(62 \%)$ out of 13 smoking patients with IPF and $3(75 \%)$ out of 4 patients with IPF and lung cancer, in whom CEA/Alb ratios in BAL fluid exceeded 2SD above the mean of the nonsmoking control patients.

In contrast, studies of sarcoidosis and hypersensitivity pneumonitis showed rather low values of CEA/Alb ratio in BAL fluid. These results may indicate that alveolitis or inflammatory reaction of lower respiratory tract characterized by high accumulation of lymphocytes in alverolar lining fluid does not cause the increase of CEA in BAL fluid.

The reason for high CEA values in BAL fluid in a subgroup of smoking control patients, patients with IPF and primary lung cancer are not clear, but some explanations may be possible. Pascal and his colleagues identified CEA in the early stage of bronchial carcinoma (carcinoma in situ) by immunohistologic techniques ${ }^{15}$. Lindgren and his colleagues studied the relationship of tissue CEA positivity detected by the immunohistological method and preinvasive lesions of the uterine cervix, and found that the CEA positivity increase with the severity of the lesions, namely, $24 \%$ in mild dysplasia, $37 \%$ in severe dysplasia and $60 \%$ in carcinoma in situ ${ }^{16)}$. Thus increase of CEA in BAL fluid may reflect histological disorders of the lower respiratory tract of these patients such as metaplasia or atypical proliferations of epithelial cells, which was frequently observed in the lower respiratory tract of the smokers ${ }^{17,18)}$, the patients with $\mathrm{IPF}^{2-4)}$ and primary lung cancer ${ }^{17}$.

Weitzman and his colleagues studied the relationship between chronic inflammation and carcinogenesis, and demonstrated that inflammatory phagocytic cells (neutrophils) can induce malignant transformation by the production of oxygen metabolites ${ }^{19-21)}$. Neutrophils are a characteristic feature of the alveolitis of $\operatorname{IPF}^{22-24)}$, as suggested by high proportion of neutrophils in BAL fluid. It is likely that neutrophils mediated parenchymal injury by secreting oxygen metabolites as well as various enzymes in IPF ${ }^{25)}$. In addition, cigarette smoke displays a wide range of oxygen radical mediated activities ${ }^{26)}$, and also attracts neutrophils to the human lung by stimulating alveolar macrophage to release a potent chemotactic factor for neutrophils ${ }^{27)}$. In this regard, it may be possible that these chronic inflammatory processes would induce cellular transformation and augumentation of CEA shedding, although the cellular events linking CEA to transformation remains unclear and we failed to show definite correlation between the CEA/Alb ratio and percentage of polymorphonuclear leukocytes in BAL fluid in the IPF patients and smoking control patients. It thus appears that the increase of CEA in epithelial lining fluid is possibly an early biochemical aberration of these transformed cells.

CEA/Alb ratio of BAL fluid was significantly higher than that of serum, therefore it is likely that virtually all the CEA found in BAL fluid is derived from pulmonary tissue, as suggested by Merill and his colleagues?).

However, it is well-known that normal lung tissue contains a large amounts of NCA which is partially reactive with CEA. Therefore, there is a possibility that NCA could be released into the lower respiratory tract in the process of lung destruction, although previous workers did not detail to this point. We proposed that the increase of CEA in BAL fluid and serum of the patients with IPF was not due to an increase of NCA by the following two reasons. First, CEA was measured by an enzyme immunoassay with highly specific monoclonal antibody, of which reaction was negligible with NCA from normal lung and spleen (personal communication to Dr. M. Kuroki, First Department of Biochemistry, School of Medicine, Fukuoka Univ., Japan, an author of the reference litrature ${ }^{12)}$ ). Second, the molecular size of CEA in BAL fluid determined by the Sephadex G-200 chromatography as shown in Fig. $3 \mathrm{~b}$ is estimated to be about two times greater than that of lung NCA.

From these points of view, we considered that an increase of CEA in BAL fluid may reflect histological disorders of the lower respiratory tract of IPF, such as metaplasia or atypical proliferation of epithelial cells. It may also indicate a greater risk of malignant change in the clinical 
course of patients with IPF.

The mechanism of the elevation of serum CEA in the patients with IPF is not yet clear. However, it is possible that CEA produced in the lung tissue is partially released into the serum, although CEA/ Alb ratios in BAL fluid did not correlate with serum CEA values.

In summary, high values of CEA/Alb ratio in BAL fluid were observed in a subgroup of the smoking control patients, patients with primary lung cancer and patients with IPF, especially who had been complicated with lung cancer. It is obvious that we need further study, but we may conclude that the increase of CEA in epithelial lining fluid is not due to NCA, and a possible marker of early histological aberration in the lower respiratory tract in these high risk patients of lung cancer.

\section{REFERENCES}

1) Turner-Warwick $M$, Lebowitz $M$, Burrows B, et al: Cryptogenic fibrosing alveolitis and lung cancer. Thorax 35: 496, 1980.

2) Fraire AE, Greenberg SD: Carcinoma and diffuse interstitial fibrosis of lung. Cancer 31: 1078, 1973.

3) Haddad R, Massaro D: Idiopathic diffuse interstitial pulmonary fibrosis (Fibrosing alveolitis), atypical epithelial proliferation and lung cancer. Am J Med 45: $211,1968$.

4) Hansen HJ, Synder JJ, Miller E, et al: Carcinoembryonic antigen (CEA) assay. Human Pathol 5: $139,1974$.

5) Blair OM, Goldenberg DM: A correlative study of bronchial cytology, bronchial washing carcinoembryonic antigen in the diagnosis of bronchogenic cancer. Acta Cytol (Baltimore) 18: 510, 1974.

6) Lemarie C, Lavandier M, Renoux M, et al: Carcinoembryonic antigen in bronchoalveolar lavage fluid (letter). N Engl J Med 303: 586, 1980.

7) Merill WW, Goodman M, Matthay RA, et al: Quantitation of carcinoembryonic antigen in the lung lining fluid of normal smokers and nonsmokers. Am Rev Respir Dis 123: 29, 1981.

8) Lowry $\mathrm{OH}$, Rosebrough NJ, Farr AL, et al: Protein measurement with Folin phenol reagent. J Biol Chem 193: 265, 1951.

9) Kleist S, Chavanel G, Burtin P: Identification of an antigen from normal human tissue that corssreacts with the carcinoembryonic antigen. Proc Nat Acad Sci 69: 2492, 1972.

10) Burtin P, Charvanel G, Hirsch-Marie H: Characterization of a second normal antigen that cross-reacts with CEA. J Immunol 111: 1926, 1973.

11) Shively JE, Todd CW, Go VLW, et al: Amino- terminal sequence of carcinoembryonic antigenlike glycoprotein isolated from colonic lavage of healthy individuals. Cancer Res 38: 503, 1978.

12) Kuroki M, Koga $Y$, Matsuoka $Y$ : Purification and characterization of carcinoembryonic antigenrelated antigen in normal adult feces. Cancer Res 41: $713,1981$.

13) Spain DM: The association of terminal bronchiolar carcinoma with chronic interstitial inflammation and fibrosis of the lungs. Am Rev Tuberc 76: 559, 1957.

14) Meyer EC, Liebow AA: Relationship of interstitial pneumonia honeycombing and atypical epithelial proliferation to cancer of the lung. Cancer 18: 322, 1965.

15) Pascal RR, Mesa-Tejada R, Bennett SJ, et al: Carcinoembryonic antigen, immunohistologic identification in invasive and intraepithelial carcinomas of the lung. Arch Pathol Lab Med 101 : 568, 1977.

16) Lindgren J, Wahlstrom T, Seppala M: Tissue CEA in premalignant epithelial lesions and epidermoid carcinoma of the uterine cervix. Int $\mathrm{J}$ Cancer 23: $448,1979$.

17) Auerbach O, Stout AP, Hammond EC, et al: Changes in bronchial epithelium in relation to smoking and in relation to lung cancer. New Engl J Med 265: 253, 1960.

18) Ford DK, Fidler HK, Lock DR: Dysplastic lesions of bronchial tree. Cancer 14: 1226, 1961.

19) Weitzman SA, Weitberg AB, Clark EP, et al: Phagocytes as carcinogen: Malignant transformation produced by human neutrophils. Science 227: $1231,1985$.

20) Weitzman SA, Stossel TP: Effects of oxygen radical scavengers and antioxidants on phagocyte-induced mutagenesis. J Immunol 128: $2770,1982$.

21) Weitberg AB, Weitzman SA, Destrempes $M$, et al: Stimulated human phagocytes produce cytogenetic changes in cultured mammalian cells. N Engl J Med 308: 26, 1983.

22) Weinberger SE, Kelman JA, Elson NA, et al: Bronchoalveolar lavage in interstitial lung disease. Ann Intern Med 89: 459, 1978.

23) Hunninghake GW, Kawanami O, Ferrans VJ, et al: Characterization of the inflammatory and immune effector cells in the lung parenchyma of patients with interstitial lung disease 123: 407, 1981.

24) Haslam PL, Turton CWG, Heard B, et al: Bronchoalveolar lavage in pulmonary fibrosis: composition of cells obtained with lung biopsy and clinical features. Thorax 35: 9, 1980.

25) Hunninghake GW, Gadek JE, Lawley TJ, et al: Mechanism of neutrophil accumulation in the lungs of patients with idiopathic pulmonary fibrosis. J Clin Invest 68: 259, 1981.

26) Stedman RL: The chemical composition of tobacco and tobacco smoke. Chem Rev 58: 153, 1968. 